\title{
Challenges in the analysis of complex systems: introduction and overview
}

\author{
Harold M. Hastings ${ }^{1,2, a}$, Jörn Davidsen ${ }^{3}$, and Henry Leung ${ }^{4}$ \\ ${ }^{1}$ Division of Science, Mathematics and Computing, Bard College at Simon's Rock, \\ Great Barrington, MA, USA \\ ${ }^{2}$ Department of Physics and Astronomy, Hofstra University, Hempstead, NY, USA \\ ${ }^{3}$ Complexity Science Group, Department of Physics and Astronomy, University of \\ Calgary, Calgary, AB, Canada \\ ${ }^{4}$ Department of Electrical and Computer Engineering, University of Calgary, Calgary, \\ $\mathrm{AB}$, Canada
}

Received 28 November 2017

Published online 28 December 2017

\begin{abstract}
One of the main challenges of modern physics is to provide a systematic understanding of systems far from equilibrium exhibiting emergent behavior. Prominent examples of such complex systems include, but are not limited to the cardiac electrical system, the brain, the power grid, social systems, material failure and earthquakes, and the climate system. Due to the technological advances over the last decade, the amount of observations and data available to characterize complex systems and their dynamics, as well as the capability to process that data, has increased substantially. The present issue discusses a cross section of the current research on complex systems, with a focus on novel experimental and data-driven approaches to complex systems that provide the necessary platform to model the behavior of such systems.
\end{abstract}

\section{Introduction}

Physical, geophysical, chemical, living and man-made systems often show behaviors that cannot be understood by studying their building blocks or constituents to ever finer detail but that are emergent. The concept of emergence can be summarized by the statement that there exists an entity (e.g. an organism) which is more than the sum of its parts [1].

Systems showing emergence are typically considered complex. This lack of simple additivity in complex systems renders linear approaches and traditional experimental and analytic frameworks useless and has led to the new scientific field of complexity science, which uses system-level approaches. The lack of simple additivity also reflects the importance of strong nonlinearities in complex systems. Prominent examples of complex systems include seismicity [2,3], climatology [4-7], human heart dynamics [8-12] and neural signaling or biological signaling processes in general [13], with immense importance for society: Recent catastrophic earthquakes in Japan, Haiti,

\footnotetext{
a e-mail: hhastings@simons-rock.edu
} 
Italy and Indonesia (loss of life $>550000$, economical damage $>\$$ US 200 billion) and ever-increasing population density in large metropolitan areas near major active faults (e.g., Tokyo, Istanbul, San Francisco bay area) highlight the great societal importance of predicting and forecasting naturally occurring earthquakes. This is also true for earthquakes unintendedly induced by geoengineering activities, such as hydraulic fracturing $[14,15]$ - a key enabling technology for unconventional resource development in the oil and gas industry.

Another example is the brain. Understanding the relationship between structure, dynamics and function in the brain is a crucial step toward innovative solutions for brain-related diseases such as epilepsy and the goal of large-scale research projects such as the CAD $\$ 1.6$ billion Human Brain Project. Another important example is the identification of genes that interact to control cellular processes by transcriptional activation of a set of target genes - identifying their interactions is an essential task for network reverse engineering in modern systems biology. Finally, cardiac arrest is a major cause of mortality in the industrialized world, responsible for 325000 deaths annually in the US alone. These examples not only emphasize the importance of understanding complex systems but also the urgent need to tackle the associated challenges.

Among the recent advances in this direction, there are four overlapping themes we will focus on here: prediction and causality; complex networks: synchronization and communication, stability and controllably, the brain, the heart and beyond; and nonlinear dynamics and pattern formation. These themes were also at the core of the recent 14th Experimental Chaos and Complexity Conference held in Banff, Canada, in 2016, which has stimulated this special issue.

\section{Prediction and causality}

Inferring cause-effect relationships from observations is one of the fundamental challenges in natural sciences and beyond. Due to the technological advances over the last decade, the amount of observations and data available to characterize complex systems and their dynamics has increased substantially, making scientists face this challenge in many different areas. One specific example is the brain for which one can make multivariate recordings of its activity on many different levels, ranging from single neurons to extended brain regions. Several analysis techniques from information theory, statistics and related disciplines exist to estimate causal influences, interactions and connectivity from general multivariate recordings under certain assumptions; see the reviews [16,17]. The connectivity is often of particular importance since it allows one to describe the dynamics of a given system in the modern language of complex network theory and to tackle questions related to structure and functionality.

Moreover, the presence of causal influences makes the behavior of a given system typically more predictable. Indeed, improving the predictability is often used as the guiding principle to build estimators for information flow and ultimately causal relations from data as it is the case for Granger causality and transfer entropy, cf. [18]. Data-driven prediction has a long history with Kalman filters one of the earliest examples [19]. In its classical formulation, the Kalman filter and their extensions such as the ensemble Kalman filters [20] require the knowledge of the dynamical model equations governing the evolution of the system at hand. In the absence of such knowledge, nonparametric approaches to data-driven prediction are typically used. These include but are not limited to methods based on the maximum entropy principle, cf. [21], and time series graphs, cf. [22], as well as methods based on delay-coordinate embedding (the Takens embedding theorem [23]) to reconstruct the nonlinear dynamics [24-28]. 
These equation-free approaches are an indication that science may be moving into a period where equations no longer play the central role in describing dynamic systems that they have played in the last 300 years [29]. Taking it even a step further, Hamilton et al. [30] have proposed a combination of delay-coordinate embedding and ensemble Kalman filtering, which can lead to superior prediction results. As shown by Hamilton et al. [31] in this special issue, this approach also solves the challenge of separating dynamical and observational noise in time series data. From a purely data-driven approach, recent developments in ensemble-based inference and machine learning have proven useful to tackle diverse problems like transcriptional gene network inference [32] and neuronal connectivity [33]. Although these approaches seem to outperform others on a case-by-case basis, their usefulness for the study of complex systems is limited, since they usually behave as black boxes, giving little insight into the behavior of the underlying system. Another model-free and datadriven approach to determine interactions and dependencies is centered around the concept of recurrence plots (see the review [34]). In this special issue, Riedl et al. [35] discuss how this technique can be generalized such that it can be applied to spatially extended systems.

For systems that are characterized by discrete events and a point process dynamics such as spiking neurons or aftershock sequences, many of the above data-driven methods need to be adapted (cf. [36,37] and references therein) and new methods have been proposed [38-40] to infer causal influences and interactions. Yet many challenges remain. These include the correct identification of downregulating or inhibitory connections [41,42], which play a fundamental role in neurosciences, for example. Another one is the detection of causal connections and event-event triggering when complete (spatio-temporal) information is lacking as discussed by Baró and Davidsen [43] in this special issue, with direct implications in the context of rock fracture $[44,45]$.

\section{Complex networks: synchronization and communication, stability and controllably, the brain, the heart and beyond}

From interacting populations of earthquake faults to the nerve cells in the brain, many complex systems can be represented as a collection of dynamical units coupled via complex architectures. Complex network theory, a marriage of ideas and methods from statistical physics and phase transitions, nonlinear dynamics as well as graph theory, has become one of the most successful frameworks for studying this type of complex systems and has led to major advances in our recent understanding of these systems and their emergent properties, cf. [46,47].

The individual dynamics of the coupled units can often be considered oscillatory or excitatory. For example, the brain and heart are largely composed of excitable cells (neurons and cardiac cells, respectively); a cell is excitable if only a sufficiently large stimulus generates a nonlinear response (action potential in a neuron) before the system returns to its rest state. Similarly, many other biological systems and chemical systems, including the now classic Belousov-Zhabotinsky chemical reaction ([48-51], see also the recent review [52]) fall into the class of excitable media.

Neurons and cardiac cells as well as chemical reactions may, however, not only generate excitable dynamics but also oscillatory dynamics; for example the normal sinus rhythm in the heart is driven by synchronized oscillations in the sino-atrial node, which generates wave of activation propagating through the rest of the heart (a network of excitable cells). Nonlinear wave propagation is one of the hallmarks of both excitable and oscillatory media $[53,54]$ and it is often present in the form of self-sustained spiral waves, presenting a specific example of a synchronization phenomenon. 


\subsection{Synchronization and communication}

The synchronization of coupled oscillators [55] is a fascinating manifestation of selforganization that nature often uses to orchestrate essential processes of life [56]. More recently, the coexistence of localized synchronized and unsychronized regions in homogeneous systems - so-called chimera states - has drawn a significant amount of attention due to its symmetry-breaking nature [57-60].

The study of synchronization in model systems often goes hand-in-hand with experiments. Hastings et al. [61] explored synchronization in networks of analog electronic Keener [62] neurons. Keener's circuit generated a non-linear "sodium current" using saturation properties of operational amplifiers, essentially realizing the original Nagumo [63] circuit for FitzHugh-Nagumo [63,64] neurons; cf. [65]. Analog multipliers have also been generate non-linear neuronal dynamics in studies of chimeras in networks of analog electronic FitzHugh-Nagumo neurons [66] and synchronization in networks of analog electronic Hindmarsh-Rose neurons [67]. In this special issue, Ahmed et al. [68] present the results of their experimental study of synchronization in a network of electronic, analog Brockett oscillators coupled through error feedback, finding robust global synchronization.

One of the pioneering theoretical contributions to synchronization is due to Kuramoto [69] who used differential equations to describe the time evolution of oscillator phase in networks of coupled oscillators. The Kuramoto model has served as a prototype for extensive studies of synchronization in these networks, and more generally, the dynamics of networks of coupled oscillators; cf. the review [70]. In this special issue, Bonnin [71] develops a Kuramoto-style model for coupled noisy oscillators, starting from a phase and amplitude description. Bonin shows that the phase noise problem can be considered as drift-diffusion process.

Finally, ensuring security is one of the major goals in communication. One approach has been to use synchronized chaotic oscillators for transmit and receive functions [72-74]. In this special issue, Seneviratne and Leung [75] describe a novel approach to secure communications using multiple chaotic signals for modulation and demodulation. Limits on the security of cryptography $[76,77]$ led to the use of physical layer security in wireless networks [78,79], including the use of spread spectrum techniques, cf. [80]. Seneviratne and Leung use a "mixture of chaotic modulation schemes to generate chaotically modulated symbols for each subcarrier of a spread-spectrum (OFDM, orthogonal frequency domain multiplexing) transmitter. As a result, the transmitted signals are indistinguishable from noise without appropriate demodulation. At the receiver, different demodulators are combined together for the different modulation schemes for enhanced security. The high security offered by this system comes with a slight performance degradation compared to conventional OFDM communication systems.

\subsection{Stability and controllability}

One frequently asked question in the dynamics of complex systems is "How stable are the dynamics?" Can we control the system so as to keep its dynamics in a satisfactory basin of attraction or drive it out of alternative less satisfactory basins of attraction? These questions of stability and controllability are central to a wide range of systems, ranging from stability of power grids (to avoid collapses such as the recent ones in the eastern US and India, for example) to cardiac dynamics (maintaining sinus rhythm and preventing degeneration into dangerous rhythms such as ventricular fibrillation as well as driving cardiac dynamics out of dangerous rhythms as done by defibrillation) to maintaining the world's fisheries. 
While many recent papers have addressed the issues of stability and controllability in networks (e.g. [81-84]), the stability analysis of large networks began with seminal papers of Wigner [85,86] and May [87] on the eigenvalues of random matrices and thus the linear stability of large random systems (see also [88] and the recent review [89]). Under appropriate additional hypotheses [90,91], the spectral radius of a large random matrix depends upon its size, connectance (fraction of entries which are non-zero) of its underlying graph, and the size of the entries, a result known as the May-Wigner stability theorem.

However, many important large networks display non-linear dynamics suggesting that different approaches might be necessary. One approach that has been proven useful in assessing stability of attractors is "basin stability" [92], a network level, non-linear stability concept, complementing the linear stability-stability paradigm. As a consequence, in particular, Menck et al. [93] showed that eliminating "dead ends" in power grids can improve their stability.

In this special issue, Smith et al. [94] address the question of stability in human balance, a question first addressed in the context of dynamics by Collins and De Luca [95] who recognized human balance as a complex, dynamic process. Stirling and Zakynthinaki [96] studied Lyapunov stability of a quiet stance. Smith et al. studied basins of attraction for postural stability (essentially basin stability), with an aim to quantify previously subjective clinical measures. Falls in the elderly are a major cause of mortality and morbidity [97,98]; Smith et al.'s ultimate overall goal is to create a quantitative fall risk assessment tool.

\subsection{The brain, the heart and beyond}

The brain and heart have been widely studied as biological networks of excitable media, that is, media which support action potentials $[8,10]$, and more generally as dynamical systems. As a specific example, Bélair et al. [99] characterized sudden cardiac death and epilepsy as dynamical diseases, "marked by sudden changes in the qualitative dynamics of physiological processes;" see also [11,100-103].

Sudden cardiac death caused by ventricular fibrillation (VF) leads to 325000 sudden cardiac deaths per year in the US, making VF a leading cause of death in the industrialized world. Both pharmacological and interventional (implantable cardiac defibrillators) have been used in attempts to reduce sudden cardiac death due to VF. The role of dynamical systems in addressing VF is evident in Weiss et al.'s [104] description of the route from ventricular tachycardia (VT) to ventricular fibrillation as a transition to spatiotemporal chaos, though the specific nature of this transition remains to be established, cf. [105-107]. This dynamical systems-level perspective also explains failures of certain drug treatments, including increased mortality from sudden cardiac death in post-myocardial infarction patients treated with apparently promising antiarrhythmic drugs (the CAST [108] and SWORD [109] trials). Although drugs in the CAST trial sharply reduced the incidence of VT (understandable at the cell level), they sharply increased the likelihood of transition to VF (understandable at the organ as network of cells level), thus increasing mortality; see also [110-113]. More recently, Uzelac et al. [114] found that current action potential models lack the necessary coupling between voltage and calcium compared to experiments and thus fail to reproduce some key dynamics. They concluded that better models are needed to study the possible pro-arrhythmic potential of drugs and avoid failures such as the CAST and SWORD trials discussed previously. In another direction, Luther et al. [115]) have taken aim at the holy grail of low-energy defibrillation using a detailed analysis of how the structure of the cardiac electrical systems affects the dynamics of defibrillation.

The dynamical systems perspective across different scales also applies to epilepsy and more generally the brain as a whole [116]. The recent, ongoing large-scale BRAIN 
initiative [117-120] aims to understand brain dynamics at all scales, ranging from local neuronal dynamics, through mesoscale and whole brain. At the single neuron and sub-neuron scale, the BigNeuron project (http://bigneuron.org/) aims to define and advance state-of-the-art single neuron reconstruction by testing on a common open platform as many open-source, automated neuron reconstruction algorithms as possible, using very large scale, publicly available single 3D neuron image data sets acquired by several light microscopy methods [121]. At larger scales, The Allen Mouse Brain Connectivity Atlas (http://brain-map.org) is a freely available, foundational resource for structural and functional investigations into the neural circuits that support behavioral and cognitive processes in health and disease [120].

At the same time, advanced MRI techniques (functional MRI (fMRI), functional connectivity MRI and diffusion MRI imaging)) have been used to study brain function as well as structure (cf. the review [122]), though it is likely that only the interplay between structure and dynamics gives rise to function [123]. A connectivity analysis of the default mode network of the brain in a resting state found that its activity was attenuated during cognitive processing [124]. More recently, Padmanabhan et al. [125] found dynamical disease (abnormal structure and function) in the default mode network in autism. A community-wide effort to understand intrinsic brain architecture in autism, the Autism Brain Imaging Data Exchange (http://fcon_1000.projects.nitrc.org/indi/abide/) in now underway [126].

A second major brain network, the task positive network, has also been studied by advanced MRI. Here Grady et al. [127] found that default mode network engagement decreased and task positive network engagement increased with aging; see also [128]. In an analysis of brain activity in higher level tasks, Protzner et al. [129] found differences in resting-state data and functional differences between Scrabble ${ }^{\circledR}$ experts and beginners engaged in visual word recognition. Experts used brain regions associated with working memory and visual perception rather than those associated with meaning retrieval, see also [130], reminiscent of differences in brain usage between chess experts and novices [131].

Brain networks have also been explored with new tools such as algebraic topology [132-134] and more generally network science ([135] and references therein), extending graph-theoretic analysis [46]. Networks of Boolean (switching) elements offer another useful model in the study of the brain as well as other biological networks such as the genetic regulatory network. Rosin et al. [136] found chaotic, periodic and excitable dynamics in an experimental study of large autonomous (not clocked) Boolean networks implemented using field programmable gate arrays. Although the genetic regulatory network have long been studied with piecewise-linear differential equations (cf. $[137,138]$ ), networks of Boolean switching circuits can readily incorporate time delays difficult to incorporate in differential equation models. This can lead to long-term transients and chimera states [139-141].

We conclude this subsection with two observations of convergence, typical of the field of applied dynamical systems. First, causality approaches appear to be a promising tool in the diagnosis of dynamical diseases, for example, EEG-based diagnosis of Alzheimer's disease [142,143]. Secondly, Falk and Bassett [144] explored the relation between brain and social networks. In this spirit, Field et al. [145] offered an initial exploration of phase transitions in social networks.

\section{Nonlinear dynamics and pattern formation}

Emergent behavior in complex systems often comes in the form of self-organized structures or patterns [146-148]. These can range from geological spatial scales [149-151] and evolutionary time scales $[152,153]$ to lab scales [154]. One specific feature of 
emergent behavior in many systems is that it is self-similar or scale-free, i.e., an absence of characteristic scales over orders of magnitude. This is in particular true for earthquakes, which exhibit such scaling properties in space, time and sizes down to the smallest scales in natural and induced settings; see [155-157] and references therein. Despite this feature, earthquake prediction remains an important, elusive challenge [158]. One possible way forward is lab experiments on rock fracture and/or stick-slip dynamics under controlled conditions; cf. [45,159,160]. For example, Lockner and co-workers have investigated a classic stick-slip model [161] in lab experiments [162], complementing earlier work [44].

On evolutionary time scales, scientists have asked whether speciation, considered as pattern formation, can occur in the absence of selection [152,153]. This type of model, a neutral model, was introduced by Hubbell $[163,164]$ as a minimal alternative to the classical concept of ecological niches [165-167], and can be viewed [164] as complementing niche theory. As a partial answer to the question of the basis for speciation, computer experiments by Bahar and colleagues found universal dynamics in emergent sympatric clustering/speciation in a neutral fitness landscape - the system displays directed percolation dynamics [152], as well as more general clustering in an agent-based model [153].

Due to the non-equilibrium nature of emergent behavior, internal fluxes are an essential part of the dynamics. In this special issue, Skiba and Filatov [168] describe pattern formation in non-linear physical diffusion processes arising from the processes of transfer of energy generated by the corresponding external forcing and then dissipated in the medium. Rapid energy transfer in these media can lead to so-called blow-up modes characterized by a period of rapid change in a variable such as temperature in a bounded region, cf. $\mathrm{Hu}$ [169]. In order to study these processes Skiba and Filatov introduce new numerical methods in a 3D spherical shell.

The balance between external forcing and internal dissipation also plays an important role in hydrodynamic systems, especially if one considers turbulence [170]. For example, Deike et al. [171] contrasted the effects of boundary conditions in an experimental study in which irregular waves are driven toward an absorbing sloping beach opposite the wavemaker or a reflecting vertical wall. One sees a quasi-one-dimensional field of nonlinear waves in the case of an absorbing boundary and a more multidirectional wave field with the reflecting boundary. Observed scaling in this experimental model is compatible with previous theoretical analysis of Falcon et al. [172].

Pattern formation and turbulence can also occur in a quantum setting, studied in Dan Lathrop's lab, with quantum vortices in superfluid helium being one specific example [173]. The study of superfluid turbulence using microscopic particles and metastable helium molecules as tracers [174] as an experimental model provides a deep understanding of turbulence with surprisingly broad applications such as defect formation from symmetry breaking [175]. Other applications range from superfluid transport of information in turning flocks of starlings [176] to cosmology, using vortex lines in a superfluid to model the dynamics of cosmological strings $[177,178]$.

The consistent quantification of the complexity of a given pattern or system is one of the open challenges in the field. Lloyd [179] has offered three abstract tests for complexity: "1. How hard is it to describe the pattern or system? 2. How hard is it to create it? 3. What is its degree of organization?" In this special issue, Nagaraj and Balasubramanian [180] offer and compare several approaches to measuring complexity, namely entropy, compressability, and internal symmetry. In comparison with Lloyd's work, Nagaraj and Balasubramanian's measures are easier to compute. In particular, data compression has wide applications and one can measure the difficulty of compression, cf. Gersho and Gray [181]. 


\section{Summary}

As we have shown in this brief review, experimental research into the dynamics of complex and chaotic systems is a broad, active field, with many interconnections among specific areas of investigation. Present and future research are enabled by high-performance computing, "big-data-sized," multi-scale analysis (especially in the study of brain and more general network dynamics), new mathematical techniques (equation-free, data-driven modeling), and new applications of classical, abstract mathematics (e.g., algebraic topology), potentially yielding a broad range of applications of important social interest - from stabilizing the power grid, understanding earthquakes, to controlling dynamical disease of the brain and heart.

\section{References}

1. P.W. Anderson, Science 177, 393 (1972)

2. C.H. Scholz, Nature 391, 37 (1998)

3. D.L. Turcotte, Fractals and chaos in geology and geophysics (Cambridge University Press, Cambridge, UK, 1997)

4. O. Peters, C. Hertlein, K. Christensen, Phys. Rev. Lett. 88, 018701 (2001)

5. J.F. Donges, Y. Zou, N. Marwan, J. Kurths, Eur. Phys. J. Special Topics 174, 157 (2009)

6. A. Corral, A. Osso, J.E. Llebot, Nat. Phys. 6, 693 (2010)

7. E.A. Martin, M. Paczuski, J. Davidsen, Europhys. Lett. 102, 48003 (2013)

8. L. Glass, M.C. Mackey, From clocks to chaos: the rhythms of life (Princeton University Press, Princeton, NJ, USA, 1988)

9. A. Garfinkel, P.S. Chen, D.O. Walter, H.S. Karagueuzian, B. Kogan, S.J. Evans, M. Karpoukhin, C. Hwang, T. Uchida, M. Gotoh, O. Nwasokwa, J. Clin. Investig. 99, 305 (1997)

10. A.T. Winfree, The geometry of biological time (Springer, New York, 2001)

11. L. Glass, Chaos 25, 097603 (2015)

12. E.M. Cherry, F.H. Fenton, T. Krogh-Madsen, S. Luther, U. Parlitz, Chaos 27, 093701 (2017)

13. S. Kauffman, The origins of order: self organization and selection in evolution, in Spin glasses and biology, edited by D.L. Stern (World Scientific, Singapore, 1993)

14. W.L. Ellsworth, Science 341, 1225942 (2013)

15. X. Bao, D.W. Eaton, Science 2016, aag2583 (2016)

16. K. Hlaváèková-Schindler, M. Paluš, M. Vejmelka, J. Bhattacharya, Phys. Rep. 441, 1 (2007)

17. M. Timme, J. Casadiego, J. Phys. A: Math. Theor. 47, 343001 (2014)

18. L. Barnett, A.B. Barrett, A.K. Seth, Phys. Rev. Lett. 103, 238701 (2009)

19. R.E. Kalman, J. Basic Eng. 82, 35 (1960)

20. G. Evensen, Data assimilation: the ensemble Kalman filter (Springer, Heidelberg, 2009)

21. E.A. Martin, J. Hlinka, A. Meinke, F. Dechterenko, J. Tintera, I. Oliver, J. Davidsen, Sci. Rep. 7, 7062 (2017)

22. J. Runge, R.V. Donner, J. Kurths, Phys. Rev. E 91, 052909 (2015)

23. F. Takens, in Detecting strange attractors in turbulence, Lecture notes in mathematics (Springer-Verlag, Berlin, 1981), Vol. 898

24. H. Kantz, T. Schreiber, Nonlinear times series analysis (Cambridge University Press, Cambridge, UK, 2004)

25. B. Schelter, M. Winterhalder, J. Timmer, Handbook of time series analysis: recent theoretical developments and applications (John Wiley and Sons, New York, 2006)

26. G. Sugihara, R. May, H. Ye, C.H. Hsieh, E. Deyle, M. Fogarty, S. Munch, Science 338, $496(2012)$

27. D. Kugiumtzis, Phys. Rev. E 87, 062918 (2013) 
28. H. Ye, R.J. Beamish, S.M. Glaser, S.C. Grant, C.H. Hsieh, L.J. Richards, J.T. Schnute, G. Sugihara, Proc. Natl. Acad. Sci. USA 112, E1569 (2015)

29. D.L. DeAngelis, S. Yurek, Proc. Natl. Acad. Sci. USA 112, 3856 (2015)

30. F. Hamilton, T. Berry, T. Sauer, Phys. Rev. X 6, 011021 (2016)

31. F. Hamilton, T. Berry, T. Sauer, Eur. Phys. J. Special Topics 226, 3239 (2017)

32. D. Marbach, J.C. Costello, R. Küffner, N.M. Vega, R.J. Prill, D.M. Camacho, K.R. Allison, M. Kellis, J.J. Collins, G. Stolovitzky, Dream5 Consortium, Nat. Methods 9, $796(2012)$

33. D. Battaglia, I. Guyon, V. Lemaire, J. Orlandi, B. Ray, J. Soriano, Neural connectomics challenge (Springer International Publishing, Cham, 2017)

34. N. Marwan, M.C. Romano, M. Thiel, J. Kurths, Phys. Rep. 438, 237 (2007)

35. M. Riedl, N. Marwan, J. Kurths, Eur. Phys. J. Special Topics 226, 3273 (2017)

36. I. Malvestio, T. Kreuz, R.G. Andrzejak, Phys. Rev. E 96, 022203 (2017)

37. T. Berry, F. Hamilton, N. Peixoto, T. Sauer, J. Neurosci. Methods 209, 388 (2012)

38. D. Marsan, O. Lenglin, Science 319, 1076 (2008)

39. I. Zaliapin, A. Gabrielov, V. Keilis-Borok, H. Wong, Phys. Rev. Lett. 101, 018501 (2008)

40. J. Moradpour, S. Hainzl, J. Davidsen, J. Geophys. Res. 119, 5518 (2014)

41. S. Ito, M.E. Hansen, R. Heiland, A. Lumsdaine, A.M. Litke, J.M. Beggs, PLoS ONE 6, e27431 (2011)

42. J.G. Orlandi, O. Stetter, J. Soriano, T. Geisel, D. Battaglia, PLoS ONE 9, e98842 (2014)

43. J. Baró, J. Davidsen, Eur. Phys. J. Special Topics 226, 3211 (2017)

44. B.D. Thompson, R.P. Young, D.A. Lockner, J. Geophys. Res.: Solid Earth 114, 2156 (2009)

45. J. Davidsen, G. Kwiatek, E.M. Charalampidou, T. Goebel, S. Stanchits, M. Rück, G. Dresen, Phys. Rev. Lett. 119, 068501068501 (2017)

46. E. Bullmore, O. Sporns, Nat. Rev. Neurosci. 10, 186 (2009)

47. M.E.J. Newman, Networks: an introduction (Oxford University Press, Oxford, UK, 2010)

48. A.P. Belousov, The periodically acting chemical reaction and its mechanism, unpublished (1951). English translation in Oscillations and traveling waves in chemical systems, edited by R. Field, M. Burger (Wiley, New York, 1985), pp. 605-613

49. A.M. Zhabotinsky, Biofizika 9, 306 (1964)

50. R.J. Field, E. Koros, R.M. Noyes, J. Am. Chem. Soc. 94, 8649 (1972)

51. R.J. Field, R.M. Noyes, J. Chem. Phys. 60, 1877 (1974)

52. A.S. Mikhailov, G. Ertl, The Belousov-Zhabotinsky reaction, in Chemical complexity, edited by A.S. Mikhailov, G. Ertl, (Springer, Cham, 2017), pp. 89-103

53. K. Showalter, I.R. Epstein, Chaos 25, 097613 (2015)

54. C. Beta, K. Kruse, Annu. Rev. Condens. Matter Phys. 8, 239 (2017)

55. A. Pikovsky, M. Rosenblum, J. Kurths, Synchronization: a universal concept in nonlinear sciences (Cambridge University Press, Cambridge, UK, 2001)

56. A. Goldbeter, Chaos 27, 104612 (2017)

57. M.J. Panaggio, D.M. Abrams, Nonlinearity 28, R67 (2015)

58. H.W. Lau, J. Davidsen, Phys. Rev. E 94, 010204(R) (2016)

59. E. Schöll, Eur. Phys. J. Special Topics 225, 891 (2016)

60. J. Davidsen, Nat. Phys. (2017), doi:10.1038/s41567-017-0014-7

61. H.M. Hastings, O.I. Hernandez, L. Jiang, B. Lai, L. Tensen, J. Yang, Dynamics of biomimetic electronic artificial neural networks, in International conference on applications in nonlinear dynamics, edited by V. In, P. Longhini, A. Palacios (Springer, Cham, 2016), pp. 195-207

62. J.P. Keener, IEEE Trans. Syst. Man Cybern. 5, 1010 (1983)

63. J. Nagumo, S. Arimoto, S. Yoshizawa, Proc. IRE 50, 2061 (1962)

64. R. FitzHugh, Biophys. J. 1, 445 (1961)

65. E.M. Izhikevich, R. FitzHugh, Scholarpedia 1, 1349 (2006)

66. E.M.E. Arumugam, M.L. Spano, Chaos 25, 013107 (2015) 
67. E. Steur, C. Murguia, R.H. Fey, H. Nijmeijer, Int. J. Bifurc. Chaos 26, 1650111 (2016)

68. H. Ahmed, R. Ushirobira, D. Efimov, Eur. Phys. J. Special Topics 226, 3199 (2017)

69. Y. Kuramoto, Self-entrainment of a population of coupled non-linear oscillators, in International symposium on mathematical problems in theoretical physics, Lecture notes in physics, edited by H. Araki (Springer, Berlin-Heidelberg, 1975), Vol. 39, pp. 420-422

70. S.H. Strogatz, Physica D 143, 1 (2000)

71. M. Bonnin, Eur. Phys. J. Special Topics 226, 3227 (2017)

72. C.W. Wu, L.O. Chua, Int. J. Bifurc. Chaos 3, 1619 (1993)

73. T.L. Carroll, L.M. Pecora, Synchronizing chaotic circuits, in Nonlinear dynamics, Circuits, edited by T.L. Carroll, L.M. Pecora (World Scientific, Singapore, 1995), pp. 215-248

74. L. Kocarev, U. Parlitz, Phys. Rev. Lett. 74, 5028 (1995)

75. C. Seneviratne, H. Leung, Eur. Phys. J. Special Topics 226, 3287 (2017)

76. M.E. Hellman, IEEE Commun. Mag. 40, 42 (2002)

77. S.V. Kartalopoulos, IEEE Commun. Mag. 44, 146 (2006)

78. Y.S. Shiu, S.Y. Chang, H.C. Wu, S.C. Huang, H.H. Chen, IEEE Wirel. Commun. 18, $66(2011)$

79. A. Mukherjee, S.A.A. Fakoorian, J. Huang, A.L. Swindlehurst, IEEE Commun. Surv. Tutor. 16, 1550 (2014)

80. R. van Nee, R. Prasad, OFDM for wireless multimedia communication (Artech House, Inc., Norwood, MA, USA, 2000)

81. F.J. Müller, A. Schuppert, Nature 478, E4 (2011)

82. N.J. Cowan, E.J. Chastain, D.A. Vilhena, J.S. Freudenberg, C.T. Bergstrom, PLoS ONE 7, e38398 (2012)

83. Y.Y. Liu, J.J. Slotine, A.L. Barabási, Proc. Natl. Acad. Sci. USA 110, 2460 (2013)

84. M.T. Angulo, J.A. Moreno, G. Lippner, A.L. Barabási, Y.Y. Liu, J. R. Soc. Interface 14, 20160966 (2017)

85. E.P. Wigner, Ann. Math. 62, 548 (1955)

86. E.P. Wigner, Ann. Math. 67, 325 (1958)

87. R.M. May, Nature 238, 413 (1972)

88. R.M. May, Stability and complexity in model ecosystems (Princeton University Press, Princeton, NJ, USA, 2001)

89. S. Allesina, S. Tang, Popul. Ecol. 57, 63 (2015)

90. J.E. Cohen, C.M. Newman, J. Theor. Biol. 113, 153 (1985)

91. S. Geman, Ann. Probab. 14, 1318 (1986)

92. P.J. Menck, J. Heitzig, N. Marwan, J. Kurths, Nat. Phys. 9, 89 (2013)

93. P.J. Menck, J. Heitzig, J. Kurths, S.J. Schellnhuber, Nat. Commun. 5, 3969 (2014)

94. V.A. Smith, T.E. Lockhart, M.L. Spano, Eur. Phys. J. Special Topics 226, 3315 (2017)

95. J.J. Collins, C.J. De Luca, Exp. Brain Res. 95, 308 (1993)

96. J.R. Stirling, M.S. Zakynthinaki, Chaos 14, 96 (2004)

97. M.E. Tinetti, N. Engl. J. Med. 348, 42 (2003)

98. CDC, Web-based Injury Statistics Query and Reporting System (WISQARS) (2015), http://www.cdc.gov/injury/wisqars/ (accessed 2016/27/10)

99. J. Bélair, L. Glass, U. an der Heiden, J. Milton, Chaos 5, 1 (1995)

100. A. Beuter, J. Bélair, C. Labrie, Bull. Math. Biol. 55, 525 (1993)

101. M.C. Mackey, J.G. Milton, Ann. N. Y. Acad. Sci. 504, 16 (1987)

102. F.L. Da Silva, W. Blanes, S.N. Kalitzin, J. Parra, P. Suffczynski, D.N. Velis, IEEE Trans. Biomed. Eng. 50, 540 (2003)

103. F.L. Da Silva, W. Blanes, S.N. Kalitzin, J. Parra, P. Suffczynski, D.N. Velis, Epilepsia 44, $72(2003)$

104. J.N. Weiss, A. Garfinkel, H.S. Karagueuzian, Z. Qu, P.S. Chen, Circulation 99, 2819 (1999)

105. T. Cameron, J. Davidsen, Phys. Rev. E 86, 061908 (2012)

106. G. St-Yves, J. Davidsen, Phys. Rev. E 91, 032926 (2015)

107. S. Alonso, M. Bär, B. Echebarria, Rep. Prog. Phys. 79, 096601 (2016) 
108. Cardiac Arrhythmia Suppression Trial (CAST) Investigators, N. Engl. J. Med. 321, $406(1989)$

109. A.L. Waldo, A.J. Camm, P.L. Friedman, D.J. MacNeil, J.F. Pauls, B. Pitt, C.M. Pratt, P.J. Schwartz, E.P. Veltri, Lancet 348, 7 (1996)

110. D.S. Echt, P.R. Liebson, L.B. Mitchell, R.W. Peters, D. Obias-Manno, A.H. Barker, D. Arensberg, A. Baker, L. Friedman, H.L. Greene, M.L. Huther, N. Engl. J. Med. 324, 781 (1991)

111. C.M. Pratt, L.A. Moyé, Circulation 91, 245 (1995)

112. D.M. Roden, Am. J. Cardiol. 78, 12 (1996)

113. D.L. Packer, T.M. Munger, S.B. Johnson, K.T. Cragun, Pacing Clin. Electrophysiol. 20, 455 (1997)

114. I. Uzelac, Y.C. Ji, D. Hornung, J. Schröder-Scheteling, S. Luther, R.A. Gray, E.M. Cherry, F.H. Fenton, Front. Physiol. 8, 819 (2017)

115. S. Luther, F.H. Fenton, B.G. Kornreich, A. Squires, P. Bittihn, D. Hornung, M. Zabel, J. Flanders, A. Gladuli, L. Campoy, E.M. Cherry, Nature 475, 235 (2011)

116. D.R. Chialvo, Nat. Phys. 6, 744 (2010)

117. A.P. Alivisatos, M. Chun, G.M. Church, R.J. Greenspan, M.L. Roukes, R. Yuste, Neuron 74, 970 (2012)

118. J. Bardin, Nature 483, 394 (2012)

119. C. Bargmann, W. Newsome, A. Anderson, E. Brown, K. Deisseroth, J. Donoghue, P. MacLeish, E. Marder, R. Normann, J. Sanes, BRAIN 2025: a scientific vision. Brain Research through Advancing Innovative Neurotechnologies (BRAIN) Working Group Report to the Advisory Committee to the Director, NIH. Available online at: http://www.nih.gov/science/brain/2025/ (US National Institutes of Health, 2014)

120. S.W. Oh, J.A. Harris, L. Ng, B. Winslow, N. Cain, S. Mihalas, Q. Wang, C. Lau, L. Kuan, A.M. Henry, M.T. Mortrud, Nature 508, 207 (2014)

121. H. Peng, E. Meijering, G.A. Ascoli, Neuroinformatics 13, 259 (2015)

122. M.J. Lowe, K.E. Sakaie, E.B. Beall, V.D. Calhoun, D.A. Bridwell, M. Rubinov, S.M. Rao, J. Int. Neuropsychol. Soc. 22, 105 (2016)

123. A. Haimovici, E. Tagliazucchi, P. Balenzuela, D.R. Chialvo, Phys. Rev. Lett. 110, $178101(2013)$

124. M.D. Greicius, B. Krasnow, A.L. Reiss, V. Menon, Proc. Natl. Acad. Sci. USA 100, $253(2003)$

125. A. Padmanabhan, C.J. Lynch, M. Schaer, V. Menon, Biol. Psychiatry: Cogn. Neurosci. Neuroimaging 2, 476 (2017)

126. A. Di Martino, C.G. Yan, Q. Li, E. Denio, F.X. Castellanos, K. Alaerts, J.S. Anderson, M. Assaf, S.Y. Bookheimer, M. Dapretto, B. Deen, Mol. Psychiatry 19, 659 (2014)

127. C.L. Grady, A.B. Protzner, N. Kovacevic, S.C. Strother, B. Afshin-Pour, M. Wojtowicz, J.A. Anderson, N. Churchill, A.R. McIntosh, Cereb. Cortex 20, 1432 (2009)

128. H. Wang, A.R. McIntosh, N. Kovacevic, M. Karachalios, A.B. Protzner, J. Cogn. Neurosci. 28, 971 (2016)

129. A.B. Protzner, I.S. Hargreaves, J.A. Campbell, K. Myers-Stewart, S. van Hees, B.G. Goodyear, P. Sargious, P.M. Pexman, Cortex 75, 204 (2016)

130. I.S. Hargreaves, P.M. Pexman, L. Zdrazilova, P. Sargious, Memory Cogn. 40, 1 (2012)

131. M. Bilalić, R. Langner, M. Erb, W. Grodd, J. Exp. Psychol.: General 139, 728 (2010)

132. A. Sizemore, C. Giusti, D.S. Bassett, J. Complex Netw. 5, 245 (2016)

133. A. Sizemore, C Giusti, R.F. Betzel, D.S. Bassett, arXiv:1608.03520 (2016)

134. M.W. Reimann, M. Nolte, M. Scolamiero, K. Turner, R. Perin, G. Chindemi, P. Dłotko, R. Levi, K. Hess, H. Markram, Front. Comput. Neurosci. 11, 48 (2017)

135. D.S. Bassett, A.N. Khambhati, S.T. Grafton, Annu. Rev. Biomed. Eng. 19, 327 (2017), and references therein

136. D.P. Rosin, D. Rontani, D.J. Gauthier, E. Schöll, Phys. Rev. Lett. 110, 104102 (2013)

137. S.A. Kauffman, J. Theor. Biol. 22, 437 (1969)

138. L. Glass, S.A. Kauffman, J. Theor. Biol. 39, 103 (1973)

139. D.P. Rosin, D. Rontani, N.D. Haynes, E. Schöll, D.J. Gauthier, Phys. Rev. E 90, 030902 (2014) 
140. O. D'Huys, J. Lohmann, N.D. Haynes, D.J. Gauthier, Chaos 26, 094810 (2016)

141. J. Lohmann, O. D'Huys, N.D. Haynes, E. Schöll, D.J. Gauthier, Phys. Rev. E 95, $022211(2017)$

142. S. Bhat, U.R. Acharya, N. Dadmehr, H. Adeli, Eur. Neurol. 74, 202 (2015)

143. J.C. McBride, X. Zhao, N.B. Munro, G.A. Jicha, F.A. Schmitt, R.J. Kryscio, C.D. Smith, Y. Jiang, NeuroImage: Clinical 7, 258 (2015)

144. E.B. Falk, D.S. Bassett, Trends Cogn. Sci. 21, 674 (2017)

145. R.J. Field, J.A. Gallas, D. Schuldberg, Commun. Nonlinear Sci. Numer. Simul. 49, 135 (2017)

146. G. Pruessner, Self-organised criticality: theory, models and characterisation (Cambridge University Press, Cambridge, UK, 2012)

147. R. Desai, R. Kapral, Dynamics of self-organized and self-assembled structures (Cambridge University Press, Cambridge, UK, 2009)

148. M. Cross, H. Greenside, Pattern formation and dynamics in nonequilibrium systems (Cambridge University Press, Cambridge, UK, 2009)

149. D.L. Turcotte, Fractals and chaos in geology and geophysics (Cambridge University Press, Cambridge, UK, 1997)

150. L. Goehring, S.W. Morris, Phys. Today 67, 45 (2014)

151. A. Farough, D.E. Moore, D.A. Lockner, R.P. Lowell, Geochem. Geophys. Geosyst. 17, $44(2016)$

152. A.D. Scott, D.M. King, N. Marić, S. Bahar, Europhys. Lett. 102, 68003 (2013)

153. D.M. King, A.D. Scott, S. Bahar, R. Soc. Open Sci. 4, 170005 (2017)

154. P. Ball, The self-made tapestry: pattern formation in nature (Oxford University Press, Oxford, UK, 2003)

155. J. Davidsen, G. Kwiatek, Phys. Rev. Lett. 110, 068501 (2013)

156. S. Maghsoudi, D.W. Eaton, J. Davidsen, Geophys. Res. Lett. 43, 10672 (2016)

157. J. Davidsen, M. Baiesi, Phys. Rev. E 94, 022314 (2016)

158. S.E. Hough, Predicting the unpredictable: the tumultuous science of earthquake prediction (Princeton University Press, Princeton, NJ, USA, 2016)

159. M.L. Blanpied, D.A. Lockner, J.D. Byerlee, J. Geophys. Res.: Solid Earth 100, 13045 (1995)

160. S.D. Goodfellow, M.H.B. Nasseri, S.C. Maxwell, R.P. Young, Geophys. Res. Lett. 49, 3179 (2015)

161. W.F. Brace, J.D. Byerlee, Science 153, 990 (1966)

162. B.D. Kilgore, A. McGarr, N.M. Beeler, D.A. Lockner, Earthquake source properties from instrumented laboratory stick-slip, in Fault zone dynamic processes: evolution of fault properties during seismic rupture, edited by M. Thomas, H. Bhat, T. Mitchell (American Geophysical Union, Washington, DC, USA, 2017), pp. 150-169

163. S.P. Hubbell, The unified neutral theory of biodiversity and biogeography (Princeton University Press, Princeton, NJ, 2001)

164. S.P. Hubbell, Funct. Ecol. 19, 166 (2005)

165. J. Grinnell, Auk 34, 427 (1917)

166. G.E. Hutchinson, Cold Spring Harb. Symp. Quant. Biol. 22, 415 (1957)

167. G.E. Hutchinson, Am. Nat. 93, 145 (1959)

168. Y.M. Skiba, D.M. Filatov, Eur. Phys. J. Special Topics 226, 3303 (2017)

169. B. Hu, in Blow-up theories for semilinear parabolic equations, Lecture notes in mathematics (Springer-Verlag, Berlin-Heidelberg, 2011), Vol. 2018

170. U. Frisch, Turbulence: the legacy of A.N. Kolmogorov (Cambridge University Press, Cambridge, UK, 2006)

171. L. Deike, B. Miquel, P. Gutiérrez, T. Jamin, B. Semin, M. Berhanu, E. Falcon, F. Bonnefoy, J. Fluid Mech. 781, 196 (2015)

172. E. Falcon, S. Fauve, C. Laroche, Phys. Rev. Lett. 98, 154501 (2007)

173. M.S. Paoletti, R.B. Fiorito, K.R. Sreenivasan, D. Lathrop, J. Phys. Soc. Jpn. 77, 111007 (2008)

174. W. Guo, M. La Mantia, D.P. Lathrop, S.W. Van Sciver, Proc. Natl. Acad. Sci. USA 111(Suppl. 1), 4653 (2014) 
175. A. Del Campo, W.H. Zurek, Int. J. Mod. Phys. A 29, 1430018 (2014)

176. A. Attanasi, A. Cavagna, L. Del Castello, I. Giardina, T.S. Grigera, A. Jelić, S. Melillo, L. Parisi, O. Pohl, E. Shen, M. Viale, arXiv:1303.7097 (2013)

177. W.H. Zurek, Nature 317, 505 (1985)

178. P.C. Hendry, N.S. Lawson, R.A.M. Lee, P.V. McClintock, C.D.H. Williams, Nature 368, 315 (1994)

179. S. Lloyd, IEEE Control Syst. Mag. 21, 7 (2001)

180. N. Nagaraj, K. Balasubramanian, Eur. Phys. J. Special Topics 226, 3251 (2017)

181. A. Gersho, R.M. Gray, in Vector quantization and signal compression (Springer Science \& Business Media, Medford, MA, USA, 2012), Vol. 159 